\title{
Fault Residual Generation via Nonlinear Analytical Redundancy
}

\author{
Martin L. Leuschen, Ian D. Walker, and Joseph R. Cavallaro
}

\begin{abstract}
Fault detection is critical in many applications, and analytical redundancy (AR) has been the key underlying tool for many approaches to fault detection. However, the conventional AR approach is formally limited to linear systems. In this brief, we exploit the structure of nonlinear geometric control theory to derive a new nonlinear analytical redundancy (NLAR) framework. The NLAR technique is applicable to affine systems and is seen to be a natural extension of linear AR. The NLAR structure introduced in this brief is tailored toward practical applications. Via an example of robot fault detection, we show the considerable improvement in performance generated by the approach compared with the traditional linear AR approach.
\end{abstract}

Index Terms-Fault detection, nonlinear systems, residuals, robotics.

\section{INTRODUCTION}

A NALYTICAL REDUNDANCY (AR) [1], [20] is a faultdetection method that allows the explicit derivation of the maximum possible number of linearly independent system model-based consistency tests for a system. Using a linear model of the system of interest, AR exploits the null-space of the state-space observability matrix to allow the creation of a set of test residuals [1]. These residuals use sensor data histories and known control inputs to detect any deviation from the static or dynamic behaviors of the model in real time.

The standard AR failure detection technique [1] is only defined for linear systems. As AR is a model-based technique, it is extremely sensitive to differences between the nominal model behavior and the actual system behavior. A system model with strong nonlinear characteristics, such as a multijoint robot manipulator, suffers considerably from linearization. The dynamics of many systems in nature are dominated by nonlinear phenomena. This makes effective implementation of the AR technique difficult, as modeling errors will generate significant false error signals when linear AR is applied.

To address this issue, in this brief, we exploit recent developments in nonlinear geometric control theory [7], [14] to extend the AR principle into the nonlinear realm. Previous papers have combined linear AR with nonlinear systems [17], [21], [22], but these suffer from all the difficulties of applying linear techniques to nonlinear systems. Other researchers have used non-

Manuscript received November 30, 2002; revised June 24, 2003. Manuscript received in final form June 24, 2004. Recommended by Associate Editor G. Yen. This work was supported in part by NASA under Grant NAG5-9785, in part by the National Science Foundation/EPSCoR under Grant EPS-9630167, and in part by the Department of Energy under Contract DE-FG07-97ER 14830.

M. L. Leuschen is with the Nomadics Incorporated, Stillwater, OK 74074 USA (e-mail: mleuschen@nomadics.com).

I. D. Walker is with the Electrical and Computer Engineering Department, Clemson University, Clemson, SC 29634 USA (e-mail: ianw@ ces.clemson. edu).

J. R. Cavallaro is with the Electrical and Computer Engineering Department, Rice University, Houston, TX 77005 USA (e-mail: cavallar@ rice.edu).

Digital Object Identifier 10.1109/TCST.2004.839577 linear observers [5] in conjunction with nonlinear observability. Although observers and AR have strong connections [13], the approach in [5] is limited to known, well-modeled, faults.

Isidori and De Persis use nonlinear parity techniques analogous to the nonlinear analytical redundancy (NLAR) technique introduced in [16]. However, the method of obtaining the unobservability space in [16] differs considerably from the original Chow-Willsky technique [1]. The approach introduced in this brief preserves the structure of the polynomial parity vector approach of the original work [1], [15]. Additionally, the work in [16] derives residuals which approximate the fault signals. The NLAR approach introduced in this brief requires minimal assumptions on the structure of the faults, emphasizing compact detection of more general system failures.

Our NLAR technique is generally applicable to systems described by nonlinear affine equations such as appropriate for robotic systems [9], [11], [12], [20]. NLAR preserves the desirable formal guarantees that are generated by AR: the resulting tests arise from spanning an observation subspace, and represent a minimal span of that space. NLAR also generates considerable improvement in performance over linear AR when performing failure detection on nonlinear systems.

Notice that in this brief, as in [1], we are not seeking to analyze the noise to develop thresholds and analyze robustness (though this is discussed in the example in Section III), but to expose the underlying structure in the (nonlinear) system which can be used to develop the residuals around which to develop robust fault detection. Dynamic fault detection systems that monitor for faults in real time can contribute significantly to system reliability [2], [3], [6], [9]-[11], [18]-[20]. Timely detection of faults can prevent the damage caused by out of control systems to both themselves and their environments [19], [20].

The brief is organized as follows. The next section introduces and details the new NLAR technique. Section III presents the application of the new technique to a robot failure detection example. Conclusions are presented in Section IV.

\section{NONLINEAR ANALYTICAL REDUNDANCY}

The core notion of analytical redundancy is both intuitive and elegant [1]. Intuition arises since AR exploits the basic concept of observability, namely, that the key information which can be learned about the model-based behavior of a system can be inferred from the observation space. Elegance follows from the processing of that information in such a way as to generate a formally complete set of residual tests: AR residuals are guaranteed both to be linearly independent and to test for all detectable deviations from the system model. Thus, every residual contains at least some information not contained in other residuals, and every observable deviation from the system model is accounted for by at least one of the AR residuals. For excellent descriptions of the technique see [1] and [4]. 
The following introduces a new method for deriving NLAR test residuals. These NLAR residuals maintain the linear AR guarantees that the residuals will span the entire observable fault space and will do so with the minimal number of residuals. The linear analytical redundancy equation [1] will be replaced with a nonlinear equation that is appropriate in the context of nonlinear observability [7].

Nonlinear systems in general are difficult to deal with analytically [7], [8], [14]. They lack the convenient structure of linear systems that has been exploited over the years. Nonlinear systems do not obey the superposition principle and can exhibit behaviors such as finite escape times, multiple equilibria, or chaos, that are quite difficult to handle appropriately with linearized control systems [8]. Observability, and by extension analytical redundancy, are made much more difficult as a result of these properties. We begin the derivation of NLAR by reviewing key existing nonlinear observability structure and results.

\section{A. Nonlinear Observability}

We consider the following nonlinear state-space control system model with $n$ states, $q$ control inputs, and $m$ sensors:

$$
\begin{aligned}
& \underline{\dot{x}}(t)=f(\underline{x}(t))+g(\underline{x}(t)) \cdot \underline{u}(t)+W(\underline{x}, t)+F(\underline{x}, t) \\
& \underline{y}(t)=h(\underline{x}(t))+V(\underline{x}, t) .
\end{aligned}
$$

Here $\underline{x}(t)$ is the state vector, $\underline{y}(t)$ is the output vector, $\underline{u}(t)$ is the input vector, $W$ represents modeling error and system disturbances, $F$ represents the fault signal(s), and $V$ represents sensor noise. These vectors represent the unmodelable differences between the physical system and the putative control model. NLAR works by eliminating everything but these unmodelable effects from the data (nonzero values of these effects lead to nonzero values of the NLAR fault detection residuals), thus, these variables can be left out of the following derivation of the model-based NLAR residuals without loss of generality.

Nonlinear systems theory includes a notion of local observability applicable to nonlinear systems that are relatively smooth [7], [14]. The local nature of the analysis restricts observability to a region of the workspace "near" the current state, an open neighborhood around the current state where the observability is valid. For practical systems, this means that the sampling rate should be high enough that the system does not invalidate the current local model over the time needed to determine the observability. This is not particularly restrictive in the case of analytical redundancy, as the approach uses relatively short sensor and control histories as necessary to approximate the derivatives.

The smoothness requirements on nonlinear observability are discussed fully in [7]. For the purposes of this brief, it will suffice to say that the system must evolve on a manifold or that any local area of the workspace should be smooth in the classical sense. This smoothness requirement is more restrictive than the local nature of the observability. Real system behaviors that are not smooth, such as Coulomb friction, are not uncommon. However, there are many important systems demonstrating smooth nonlinearities that this nonlinear notion of observability can directly apply to. For example, in a hydraulic system as examined in [10] and [12], the nonlinearity is in the form of the square root.
TABLE I

RELATIONSHIP BETWEEN LINEAR AND NONLINEAR OBSERVABILITY SPACES

\begin{tabular}{c|c|c}
\hline Linear Value & Linear Notation & Nonlinear Equivalent \\
\hline$\left[C \underline{x}, C A \underline{x}, C A^{2} \underline{x}, C A^{3} \underline{x}, \ldots\right]^{T}$ & $O \underline{x}$ & $\mathcal{O}$ \\
\hline$\left[C, C A, C A^{2}, C A^{3}, \ldots\right]^{T}$ & $O$ & $\nabla \mathcal{O}$ \\
\hline
\end{tabular}

This is obviously smooth in the classical sense. The new NLAR technique introduced in this brief using the Isidori formulation of nonlinear observability is clearly appropriate for dealing with such systems.

According to the notion of observability in [7], a system is locally observable if

$$
\operatorname{Rank}(\nabla \mathcal{O})=n
$$

where: $\mathcal{O}=\operatorname{span}\left\{h_{j}, L_{k_{1}} L_{k_{2}} \ldots L_{k_{p}} h_{j}\right\}, j=1, \ldots, m p=$ $1,2, \ldots, k_{i} \in \operatorname{span}\left\{f, g_{1}, \ldots, g_{q}\right\}$, and

$$
L_{\underline{k}} h=\sum_{i=1}^{n} \frac{\partial h(\underline{x})}{\partial x_{i}} k_{i}(\underline{x})=\langle\nabla(h(\underline{x})), \underline{k}(\underline{x})\rangle
$$

is the Lie derivative of scalar function $h$ in the direction of vector field $\underline{k}$ using the standard inner product $\langle$,$\rangle . Recursed Lie$ derivatives, as seen in the definition of $\mathcal{O}$, are written in one of the following ways: $L_{\underline{i}}\left(L_{\underline{j}}\left(L_{\underline{k}} h\right)\right)=L_{\underline{i}} L_{\underline{j}} L_{\underline{k}} h=L_{\underline{i} \underline{j}} h$. It is common to omit the vector notation for the subscript, as: $L_{f} h$. The requirement $\operatorname{Rank}(\nabla \mathcal{O})=n$ is identical to the well-known linear observability criterion, based on the linear observability matrix $O$. Note that the linear $O$ and nonlinear $\mathcal{O}$ are equivalent concepts of observability but not equivalent to each other technically. $O \underline{x}$ corresponds to $\mathcal{O}$ and $O$ to $\nabla \mathcal{O}$. This can be somewhat confusing, but these notations are established [1], [7]. Table I clarifies this relationship.

However, although a valid notion of nonlinear observability appears to be a necessary prerequisite for nonlinear AR, it is not a sufficient one. One needs to be able to determine the nullspaces required by the AR equation [1] where the canonical AR equation is expressed as $\Omega^{\perp} O_{D D}=R=[0]$. Linear observability has properties exploited in AR do not easily transfer to the nonlinear case. The more complex dependence of the control system elements on the state prevents the construction of the key quantity similar to $O_{D D}$ in AR by trivial anology. Isolating the state requires some innovative manipulations, such as the techniques for overcoming these stumbling blocks that are the core innovations of this brief. These novel techniques are introduced in the following subsections.

\section{B. Nonlinear Annihilator}

The obvious approach to converting the $\mathcal{O}$ notion of observability for AR use would be to follow the linear AR method as closely as possible. This means we need to rederive the elements of the canonical AR equation $\Omega^{\perp} O_{D D}=R=[0]$ for the nonlinear system [1]. The obvious approach of stacking the elements of the $\nabla \mathcal{O}$ to create a matrix analogous to the linear $O$ matrix $\mathcal{O}_{M}$ and finding its left null space $\left(\Omega^{\perp}\right)$, as seen in (3), has some serious problems

$$
\Omega^{\perp} \nabla \mathcal{O}_{M}=[0]
$$


where

$$
\nabla \mathcal{O}_{M}=\nabla\left[\begin{array}{c}
\vdots \\
h_{j} \\
\vdots \\
L_{k_{i}} h_{j} \\
\vdots \\
L_{k_{i}} L_{k_{l}} h_{j} \\
\vdots
\end{array}\right]
$$

and $j=1, \ldots, m,\{i, l, p\}=1,2, \ldots, k_{i} \in$ $\operatorname{span}\left\{f, g_{1}, \ldots, g_{q}\right\}$, and $[0]$ is the null matrix. This approach does not directly lead to any useful AR relations. The main cause of this is the lack of useful alternate formulations of the observability which can be used as annihilators. Therefore, we develop the novel grouped formulation as expressed in (4), which yields a much more tractable analysis of the observability for the purposes of NLAR. We refer to this as $\mathcal{O}_{\triangle}$, expressed in (4). This approach sums the elements of $\mathcal{O}$ that are Lie differentiated to the same degree. $\mathcal{O}_{\triangle}$ is roughly triangular in form when written out and a vital component of NLAR

$$
\mathcal{O}_{\triangle}=\left[\begin{array}{c}
h(\underline{x}(t)) \\
\sum_{j=0}^{q} L(j) \\
\sum_{l=0}^{q} \sum_{j=0}^{q} L(j, l) \\
\sum_{m=0}^{q} \sum_{l=0}^{q} \sum_{j=0}^{q} L(j, l, m) \\
\vdots
\end{array}\right]
$$

where

$$
\begin{aligned}
L(j, l, m, \ldots) & =\left(u_{j} u_{l} u_{m} \ldots\right) L_{\ldots k(m) k(l) k(j)} h(\underline{x}(t)) \\
u_{0} & =1, \quad k(j)=\left\{\begin{array}{ll}
f, & j=0 \\
g_{j}, & j \neq 0
\end{array} .\right.
\end{aligned}
$$

$\mathcal{O}_{\triangle}$ will be used to (algebraically) generate a matrix $\Omega^{\perp}$ such that $\Omega^{\perp} \mathcal{O}_{\triangle}=0$, the canonical AR equation. Note that this formulation appears to suppress information available in the observation space by combining the elements of $\mathcal{O}$ that share the same degree of Lie differentiation. Basically, instead of considering each element of $\mathcal{O}$ individually, we choose to take the linear combination of those elements corresponding to the way they combine to form the sensor measurements and their derivations. Therefore, in some cases, this might lead to supressing some information available in the observation space. Fortunately, this will seldom be an issue in real systems. In sensitive systems where NLAR analysis is appropriate, such as aerospace and robotic systems, thorough instrumentation of the system is common, and the restriction implied by (4) is not likely to restrict the observability space ultimately computed for the system. $\nabla \mathcal{O}_{\triangle}$ is identical to the linear observability $O$ for a linear system, as all the gradients of Lie derivatives with respect to $g$ are zero in the linear case.

\section{Incorporation of Input-Output Information $\left(\mathcal{O}_{\triangle D D}\right)$}

Next, given the form of (4), we reformulate $\mathcal{O}_{\triangle}$ as $\mathcal{O}_{\triangle D D}$, in terms of control inputs $u_{i}$ and sensor readings $y_{i}$ to complete the NLAR parity equation $\Omega^{\perp} \mathcal{O}_{\triangle D D}$. Initially, it is assumed that the sensor function $h(\underline{x}(t))$ is linear and represented by $C \cdot \underline{x}(t)$ and that there is a single input $u$ (neither assumption is strictly necessary, and this is done for notational convenience here). Noting the stacked time derivatives of $y$

$$
\begin{aligned}
& {\left[\begin{array}{c}
\underline{y}(t) \\
\dot{y}(t) \\
\underline{\ddot{y}}(t) \\
\vdots
\end{array}\right]=\left[\begin{array}{c}
C \cdot \underline{x}(t) \\
\frac{d}{d t} C \cdot \underline{x}(t) \\
\frac{d^{2}}{d t^{2}} C \cdot \underline{x}(t) \\
\vdots
\end{array}\right]=\left[\begin{array}{c}
C \cdot \underline{x}(t) \\
C \cdot \underline{\dot{x}}(t) \\
C \cdot \underline{\ddot{x}}(t) \\
\vdots
\end{array}\right]} \\
& =\left[\begin{array}{c}
C \cdot \underline{x}(t) \\
C \cdot(f(\underline{x}(t))+g(\underline{x}(t)) \underline{u}(t)) \\
C \cdot \frac{d}{d t}(f(\underline{x}(t))+g(\underline{x}(t)) \underline{u}(t)) \\
\vdots
\end{array}\right]
\end{aligned}
$$

by recursively chaining and expanding the derivatives of $f$ and $g$ and then converting to Lie derivative form yields $\mathcal{O}_{\triangle}$

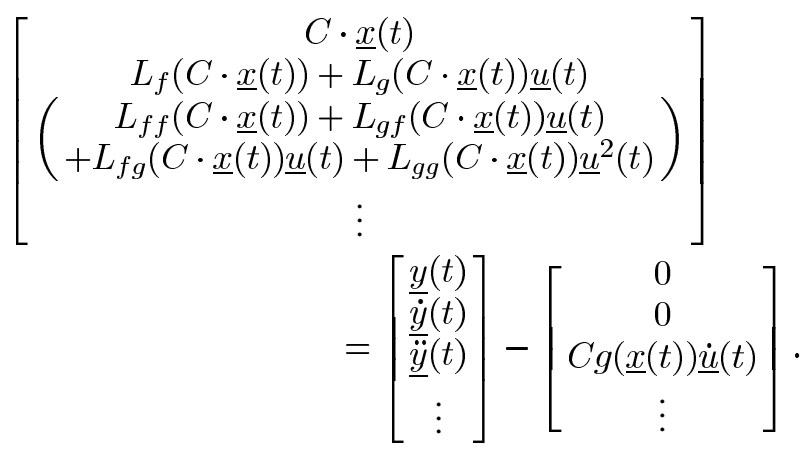

The LHS of (5) is $\mathcal{O}_{\triangle}$ for the postulated system; this is the observability-based reformulation needed for nonlinear AR. This construction can of course be repeated for multiple control inputs, and extended in a straightforward way for nonlinear sensors [9]. Equation (6), shown at the bottom of the page, gives explicit $\mathcal{O}_{\triangle D D}$ values for a generalized nonlinear system up to the third derivative. (All of the Lie derivatives of (6) are with respect to $C \cdot \underline{x}(t)$, thus, $L_{g}=L_{g}(C \cdot \underline{x}(t))=C \cdot g$.)

The number of additional terms on the RHS of (6) grows geometrically as the degree of derivation increases. Additionally, many of these RHS terms contain explicit references to the state $\underline{x}(t)$. NLAR, thus, requires that the system is observable. This

$$
\mathcal{O}_{\triangle D D}=\left[\begin{array}{c}
\frac{y}{\dot{y}}(t)-0 \\
\underline{\ddot{y}}(t)-\sum \underline{\dot{u}}(t) L_{g} \\
\underline{d}^{3} \\
\frac{d^{3}}{d t^{3}} \underline{y}(t)-\left(\begin{array}{c}
\sum \underline{\ddot{u}}_{i}(t) L_{g_{i}}+\sum \underline{\dot{u}}_{i}(t) L_{\underline{x}_{g_{i}}}+\sum \dot{\underline{u}}_{i}(t) L_{g_{i} f} \\
+\sum \underline{\underline{u}}_{i}(t) L_{f g_{i}}+\sum \underline{u}_{i}(t) \sum \underline{\dot{u}}_{j}(t) L_{g_{i} g_{j}} \\
+\sum \underline{\dot{u}}_{i}(t) \sum \underline{u}_{j}(t) L_{g_{i} g_{j}}
\end{array}\right)
\end{array}\right]
$$


TABLE II

COMPARISON OF LINEAR AND NONLINEAR AR TECHNIQUES

\begin{tabular}{l|c|c}
\hline Concept & Linear AR & Nonlinear AR \\
\hline \hline System & $\dot{x}=A \underline{x}+B u$ & $\underline{\dot{x}}=f(\underline{x})+g(\underline{x}) \cdot \underline{u}$ \\
\hline Sensors & $C \underline{x}$ & $C \underline{x}(h(\underline{x})$ possible $)$ \\
\hline Null Space & $\Omega^{\perp} O=[0]$ & $\Omega^{\perp} \mathcal{O}_{\triangle}=[0]$ \\
\hline Key Structure & $O_{D D}$ & $\mathcal{O}_{\triangle D D}$ \\
\hline Total \# of Tests & $\sum\left(r_{j}(\right.$ lin $\left.)\right)+(m-n)$ & $\sum\left(r_{j}(\right.$ nonlin $\left.)\right)+(m-1)$ \\
\hline \# of Independent Tests & $\sum\left(r_{j}(\right.$ lin $\left.)\right)+(m-n)$ & $\sum\left(r_{j}(\right.$ nonlin $\left.)\right)+(m-n)$ \\
\hline \# of Redundant Tests & 0 & $n-1$ \\
\hline
\end{tabular}

is an inevitable consequence of working with nonlinear systems, where the state independence of the linear case cannot be expected.

\section{D. $\mathcal{O}_{\triangle D D}$ and the Number of Independent NLAR Tests}

Now that both of the main elements $\left(\Omega^{\perp}\right.$ and $\left.\mathcal{O}_{\triangle D D}\right)$ of the NLAR equation have been derived, the remaining issue is to determine how many elements of $\mathcal{O}_{\triangle D D}$ must be retained to generate a minimal set of residuals. In the nonlinear case, all of the rank and span arguments of the original AR formulation are valid except for one: the Cayley-Hamilton theorem does not directly apply. Note that $\mathcal{O}_{\triangle D D}$ is a vector-valued nonlinear function, and cannot be directly checked. However, by considering $\nabla \mathcal{O}_{\triangle D D}$, we can infer the information content. It is straightforward to calculate [9] the dimension $r_{j}$ (nonlin) of the associated observation space for each sensor $j$ (from the associated rank in $\nabla \mathcal{O}_{\triangle D D}$ ), and it is easy to see that these quantities are well-defined and well-behaved. The number of residuals retained therefore corresponds to the sum of these ranks.

This is the only remaining requirement needed to reproduce the intuitive linear argument for the dimensionality of the AR residual space on the nonlinear system, and so the number of NLAR tests, $N_{\mathrm{NLAR}}=\sum_{j=1}^{m}\left(r_{j}(\right.$ nonlin $\left.)\right)+(m-n)$. As the rank of the nonlinear gradient submatrices are always greater than or equal to the ranks of the linear submatrices $\left(r_{j}(\right.$ nonlin $) \geq r_{j}($ lin $\left.)\right)$, NLAR is guaranteed to generate at least as many independent test residuals as linear AR and will usually generate more.

As the gradient of $\mathcal{O}$ is not taken explicitly in NLAR, the rank of the $\Omega^{\perp}$ matrix (constructed via $\mathcal{O}$ ) will be quite different from the linear case and NLAR residuals that are not independent will be generated. Precisely $n-1$ redundant equations will be created, as implied by the dimensions (superscripted) in

$$
\begin{aligned}
& \Omega^{\perp\left(\left(\sum\left(r_{j}+1\right)\right)-1\right) \times\left(\sum\left(r_{j}+1\right)\right)}\left[\begin{array}{c}
\vdots \\
C_{j} \\
\vdots
\end{array}\right]^{\left(\sum\left(r_{j}+1\right)\right) \times 1} \\
&=\underline{0}^{\left(\left(\sum r_{j}+1\right)-1\right) \times 1} .
\end{aligned}
$$

As eliminating the redundant equations from the valid NLAR residuals is a trivial problem (the redundant equations themselves are usually trivial), this is a minor concern.

\section{E. Assembling the Components}

We now have all the components to formally define NLAR test residuals. The formulation $\mathcal{O}_{\triangle}$ allows calculation of a left null-space $\Omega^{\perp}$ compatible with $\mathcal{O}_{\triangle D D}$. The discussion in the

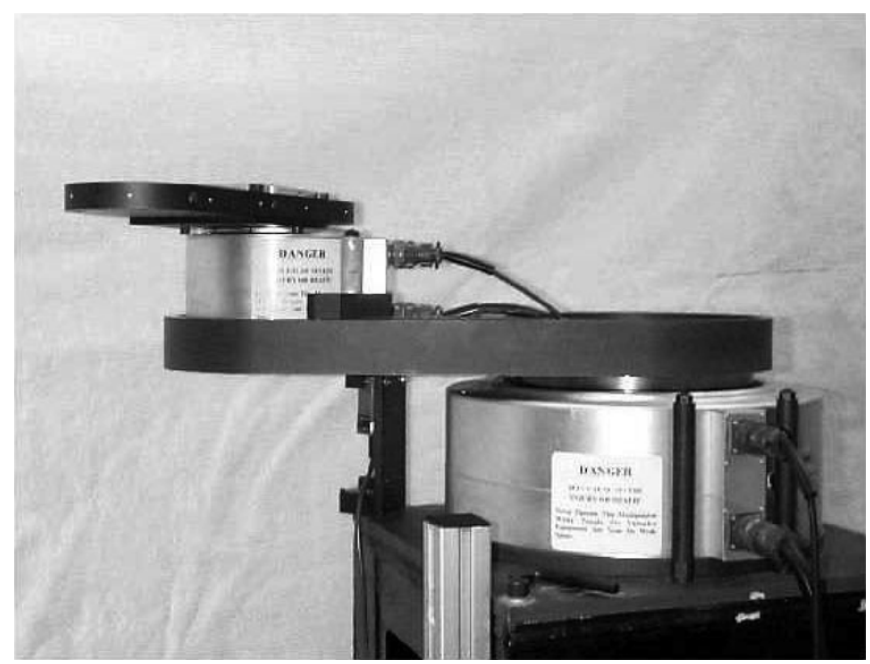

Fig. 1. Integrated Motion Inc. Robot.

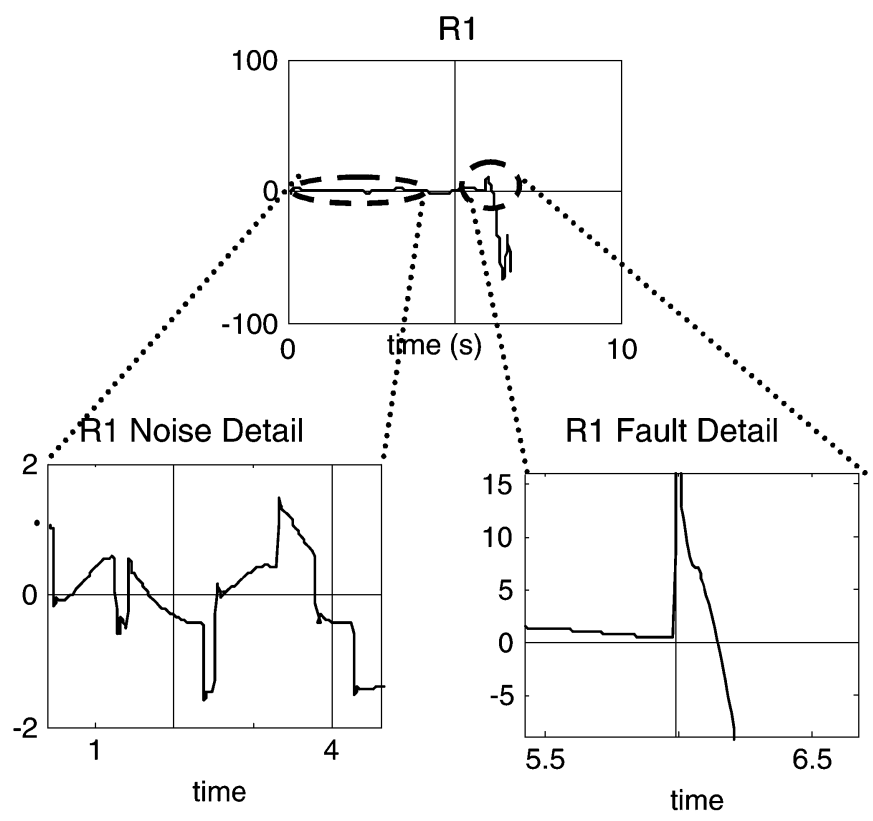

Fig. 2. Typical IMI NLAR test residual.

TABLE III

\begin{tabular}{|c|c|c|}
\hline Test Label & Description & Linear AR? \\
\hline$R_{1}$ & $\begin{array}{l}\text { Second model equation checked against shoulder re- } \\
\text { solver. Tests the acceleration of the system. }\end{array}$ & $\mathrm{Y}$ \\
\hline$R_{2}$ & $\begin{array}{l}\text { Derivative of second model equation checked against } \\
\text { shoulder resolver. Tests the jerk of the system. }\end{array}$ & $\mathrm{Y}$ \\
\hline$R_{3}$ & $\begin{array}{l}\text { Second derivative of second model equation checked } \\
\text { against shoulder resolver. Tests the derivative of the jerk. }\end{array}$ & $\mathrm{N}$ \\
\hline$R_{4}$ & $\begin{array}{l}\text { Sensor comparison of shoulder tachometer with deriva- } \\
\text { tive of shoulder resolver. }\end{array}$ & $\mathrm{Y}$ \\
\hline$R_{5}$ & Derivative of $R_{4}$ & $\mathrm{Y}$ \\
\hline$R_{6}$ & Second derivative of $R_{4}$. & $\mathrm{N}$ \\
\hline$R_{7}$ & $\begin{array}{l}\text { Fourth model equation checked against elbow resolver. } \\
\text { Tests the acceleration of the system. }\end{array}$ & $\mathrm{Y}$ \\
\hline$R_{8}$ & $\begin{array}{l}\text { Derivative of fourth model equation checked against } \\
\text { elbow resolver. Tests the jerk of the system. }\end{array}$ & $\mathrm{Y}$ \\
\hline$R_{9}$ & $\begin{array}{l}\text { Sensor comparison of elbow tachometer and derivative } \\
\text { of elbow resolver. }\end{array}$ & $\mathrm{Y}$ \\
\hline$R_{10}$ & Derivative of $R_{9}$ & $\mathrm{Y}$ \\
\hline$R_{11}$ & $\begin{array}{l}\text { Second derivative of fourth model equation checked } \\
\text { against elbow tachometer. Tests the derivative of the jerk. }\end{array}$ & $\mathrm{N}$ \\
\hline
\end{tabular}

NLAR RESIDUALS FOR IMI ROBOT 

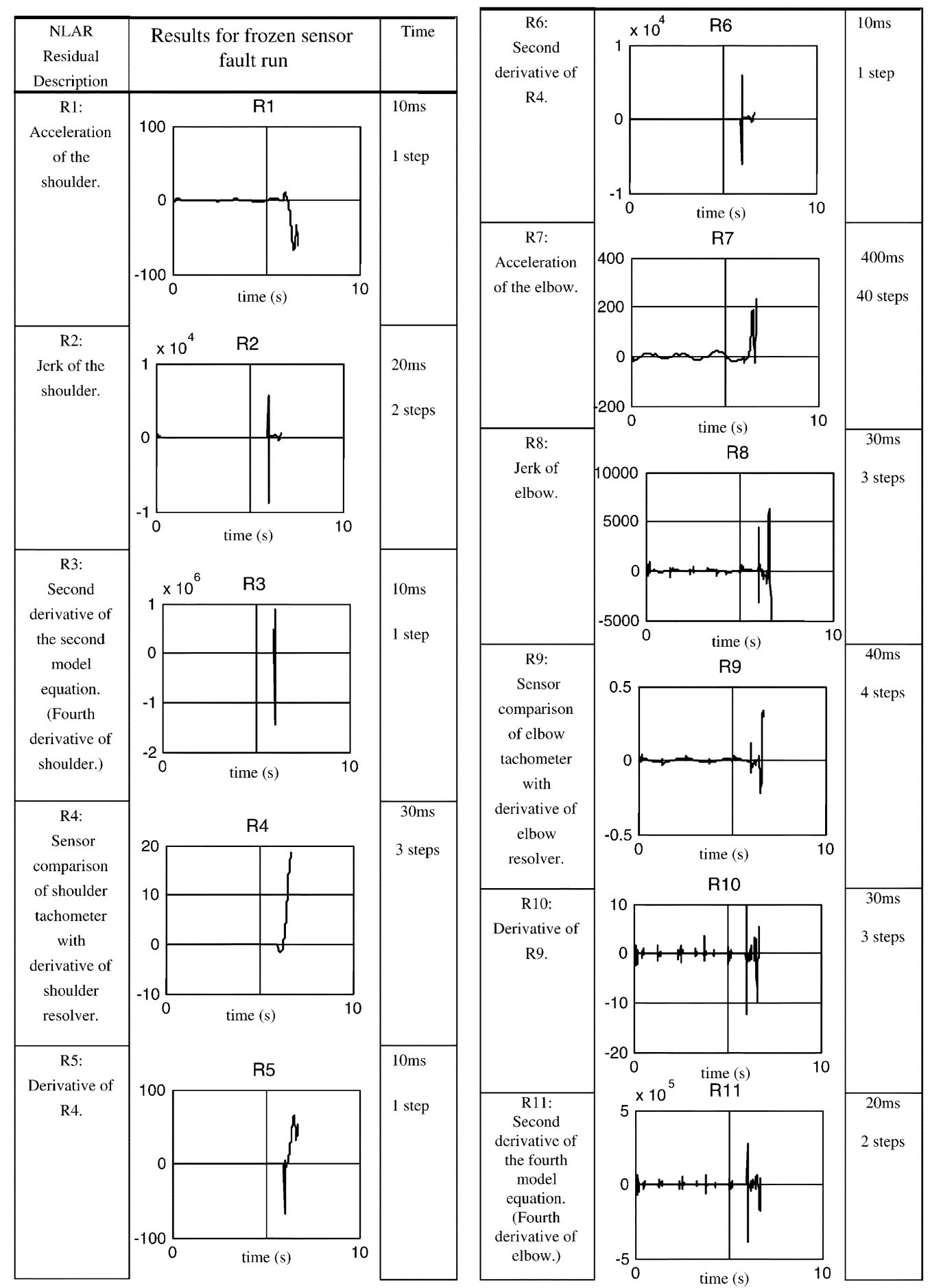

Fig. 3. IMI NLAR residuals for a frozen sensor fault.

previous subsection determines how many rows of $\mathcal{O}_{\triangle D D}$ are required and how many of the resulting residuals are independent. The full NLAR algorithm is the following.

1) Determine $\mathcal{O}_{\triangle}$ and its left null $\Omega^{\perp}$ (algebraically, from the form of $\mathcal{O}_{\triangle}$ ).

2) Determine $\mathcal{O}_{\triangle D D}$.

3) Find the rank $r_{j}$ (nonlin) of each submatrix in $\nabla \mathcal{O}_{\triangle D D}$. Keep $r_{j}$ (nonlin) +1 rows in each subvector.

4) Apply the NLAR equation: $\Omega^{\perp} \mathcal{O}_{\triangle D D}=R$, where $R$ is the vector of AR residuals

5) Use $N_{\mathrm{NLAR}}=\sum_{j=1}^{m}\left(r_{j}(\right.$ nonlin $\left.)\right)+(m-n)$ to determine how many independent residuals there are. Delete redundant residuals.

The NLAR structure is summarized in Table II. Note that implementation of the approach requires both full observability and derivatives of the components of both $y$ and $u$. This could be a serious issue for some systems (for examples, those with input discontinuities). However for many practical applications, such as in the robot example presented in the following, this is not a major problem in practice. 


\section{EXAMPLE: NLAR FOR A ROBOT MANIPULATOR}

We present an example of the NLAR technique applied to the direct drive Integrated Motor Inc. (IMI) two-joint robot manipulator (see Fig. 1), modified with an encoder and tachomoter on each joint [11]. (Implementation for a physical hydraulic actuator testbed can be found in [12].) A Simulink model of the IMI robot at $1000 \mathrm{~Hz}$ was used to examine the effect of various simulated faults on the NLAR residuals. The PID controller used only encoder feedback, with the redundant tachometers used only for sensor redundancy in the NLAR residuals. The NLAR loop was inside the controller loop, thus, the controller did not need to be considered in the NLAR residual derivation. (Analytical redundancy in general is independent of the controller, which is a useful property.) Both linear and nonlinear AR residuals were derived and tested.

The final set of 11 independent residuals described in terms of the model equations are shown in Table III. Note that while some of the intermediate steps are fairly complex, the resulting tests are various comparisons of system equations and their derivatives. These involve fewer terms, and even the highest order derivatives used are easily calculable in real time on a modern computer.

The corresponding linear AR residuals were also derived. These residuals are the linearized system equations and their various derivatives, along with sensor comparisons. The sensor comparisons are the same in the linear and nonlinear residuals, but the model-dependent AR test residuals, though conceptually equivalent to NLAR residuals, are less effective than NLAR residuals, as they are derived from a linearized model of the system. Also note that linear AR generates three fewer residuals than NLAR, which means that information is lost in the linearization. If linear AR generated a linearized version of a NLAR test, it is noted in the final column of Table III.

Faults were considered detected if the magnitude of the NLAR residual was at least twice the maximum value achieved in a fault-free run with the same parameters. More sophisticated techniques are possible, but are not developed here. The NLAR output of Fig. 2 is typical of the results. Before the fault occurs at $t=6 \mathrm{~s}$, the NLAR residuals mostly show noise-like fluctuations around a mean of 0 . These are caused by the difficulties of derivate measurement in a sampled system and unmodeled Coulomb friction. Low level noise caused similar effects in tests done on a physical hydraulic testbed in [12].

Once the fault occurs this particular NLAR test detects it on the same time step. Close examination of the fault detail on Fig. 2 shows that the residual at $t=6 \mathrm{~s}$ is about an order of magnitude larger than the fault free noise.

The table of residuals presented in Fig. 3 represents the results of the NLAR residuals for a frozen sensor fault-the shoulder resolver is frozen at its current value at $t=6 \mathrm{~s}$. As seen in the plotted results, all of the NLAR residuals detect the fault very quickly; many of them detect it on the very next time step. This is an important result, as a frozen sensor does not immediately cause significant tracking errors.

Fig. 4 is a comparison figure showing the improvement in performance of NLAR over linear AR for a broken motor fault. Note that linear AR produces eight rather than eleven residuals, and that four of these are sensor comparisons identical to the

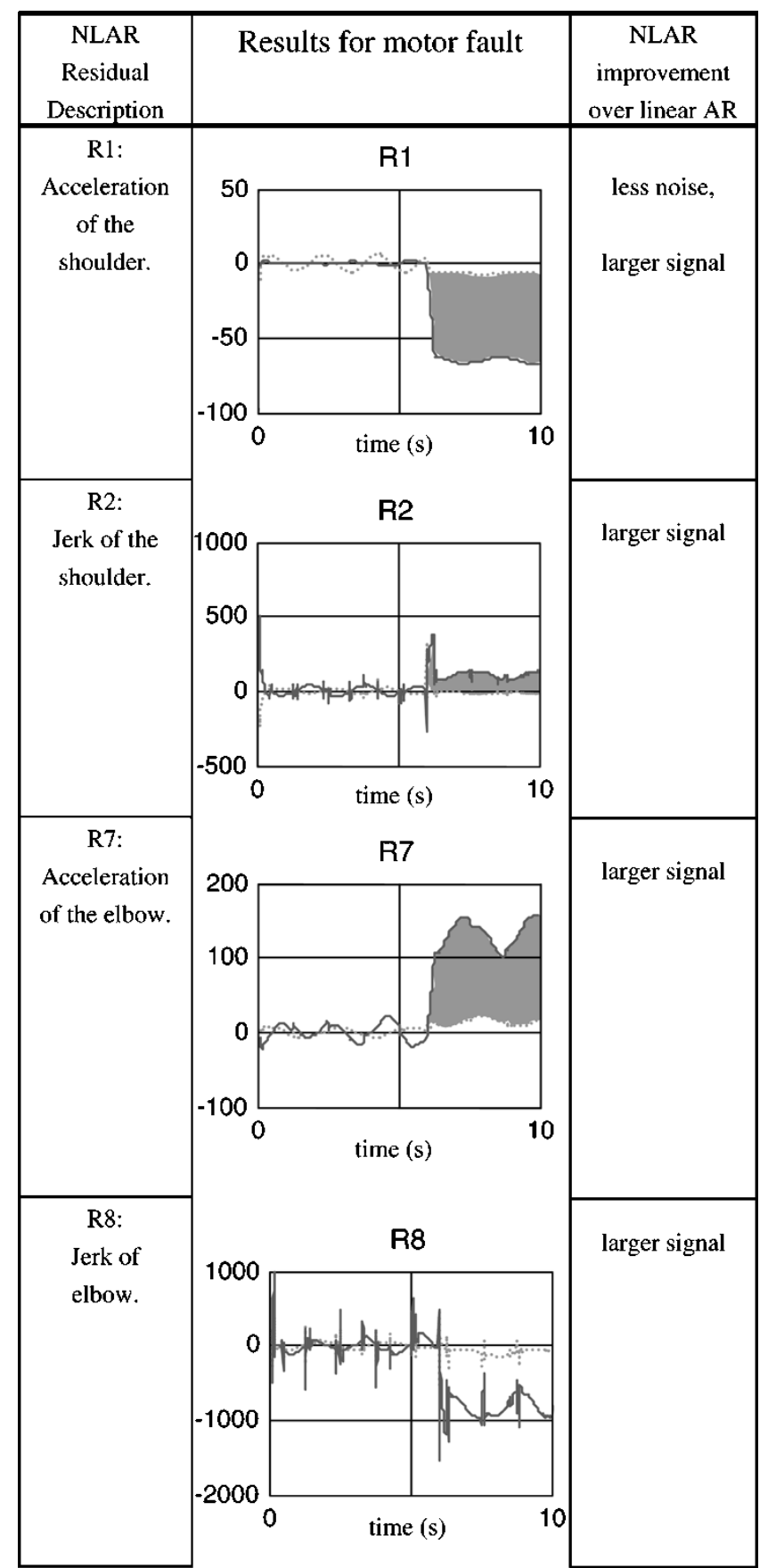

Fig. 4. IMI NLAR test result (solid dark) versus linear AR test result (dotted light) for motor fault.

NLAR residuals and therefore not shown. This leaves four tests that are analogous to NLAR residuals but linear in nature. For example, there is a linear AR test that examines the linear model of acceleration of each joint that can be compared to the NLAR test of the nonlinear, model of the same. These analogous residuals are compared in Fig. 4. It is clear that NLAR outperforms linear AR on all four of these residuals by about an order of magnitude.

\section{CONCLUSION}

The new formal NLAR fault detection technique introduced in this brief allows formal model-based failure detection for 
nonlinear systems without requiring linearizations. This is done without requiring computation beyond the abilities of easily available computer hardware. The NLAR approach is valid for the physically significant class of affine nonlinear systems, and is shown to be a generalization of the classical linear AR approach. Table II summarizes this extension. NLAR test residuals are guaranteed to detect observable deviations (exploiting observability in the nonlinear sense) from the system model, the key requirement in any fault detection system. Similar to the parallel developments for linear AR, the aim is to expose the underlying structure in the system in order to develop residuals around which to develop robust fault detection. Therefore the emphasis here is not on the next step of threshold development or fault isolation. The example demonstrates how the nonlinear method expands the linear method accurately and efficiently.

It is important to understand that this is a structural brief and it, thus, focuses providing a practical method of fault detection. Analysis of noise and modeling error is important and a planned area for future investigation. Due to the repeated derivatives, the NLAR approach is best suited to nonlinear systems that are well-modeled and relatively noiseless, with clean sensor data such as robot manipulators. For those systems, up to the present time linear AR has been the only analytical redundancy option available in practice, so NLAR is practically useful, even in its present form, for this class of relatively noiseless systems. Extensive work has been done examining the performance of the well-established linear AR method for these systems. NLAR should significantly improve on the performance of linear AR for these systems.

\section{REFERENCES}

[1] E. Y. Chow and A. S. Willsky, "Analytical redundancy and the design of robust failure detection systems," IEEE Trans. Autom. Control, vol. AC-29, no. 7, pp. 603-614, Jul. 1984.

[2] Environmental Waste Management Project Fact Sheet (1997, Oct.). [Online]. Available: http://www.fetc.doe.gov/publications/factsheets/ewm/ index.html

[3] B. S. Dhillon, Robot Reliability and Safety. New York: SpringerVerlag, 1991.

[4] J. J. Gertler, Fault Detection and Diagnosis in Engineering Systems. New York: Marcel Dekker, 1998.
[5] H. Hammouri, M. Kinnaert, and E. H. El Yaagoubi, "Observer-based approach to fault detection and isolation for nonlinear systems," IEEE Trans. Autom. Control, vol. 44, no. 10, pp. 1879-1884, Oct. 1999.

[6] R. Isermann, W. Appel, B. Freyermuth, A. Fuchs, W. Janik, D. Neumann, Th. Reiss, and P. Wanke, "Model based fault diagnosis and supervision of machines and drives," in Proc. IFAC 11th Triennial World Congr., Tallinn, USSR, Aug. 1990, pp. 1-12.

[7] A. Isidori, Nonlinear Control Systems. New York: Springer-Verlag, 1995.

[8] H. K. Khalil, Nonlinear Systems, 2nd ed. Englewood Cliffs, NJ: Prentice-Hall, 1996.

[9] M. L. Leuschen, "Derivation and application of nonlinear analytical redundancy techniques with applications to robotics," Ph.D. dissertation, Dept. Elect. Comput. Eng., Rice Univ., Houston, TX, 2001.

[10] M. L. Leuschen, I. D. Walker, and J. R. Cavallaro, "Experimental AR fault detection methods for a hydraulic robot," in Proc. ANS 9th Topical Meeting Robotics Remote Systems, Seattle, WA, Mar. 2001, p. F131.

[11] _ " "Robotic fault detection using nonlinear analytical redundancy," in Proc. IEEE Int. Conf. Robotics Automation, Washington, DC, May 2002, pp. 456-463.

[12] — , "Nonlinear Fault Detection for Hydraulic Systems," in Fault Diagnosis and Fault Tolerance for Mechatronic Systems, Recent Advances, $\mathrm{F}$. Caccavale and L. Villani, Eds, Berlin, Germany: Springer-Verlag, 2002, pp. 169-191.

[13] J. F. Magni and P. Mouyon, "On residual generation by observer and parity space approaches," IEEE Trans. Autom. Control, vol. 39, no. 2, pp. 441-447, Feb. 1994.

[14] H. Nijmeijer and A. J. Van der Schaft, Nonlinear Dynamical Control Systems. New York: Springer-Verlag, 1990.

[15] M. Nyberg and L. Nielsen, "A universal Chow-Willsky scheme and detectability criteria," IEEE Trans. Autom. Control, vol. 45, no. 1, pp. 152-156, Jan. 2000.

[16] C. De Parsis and A. Isidori, "A geometric approach to nonlinear fault detection and isolation," IEEE Trans. Autom. Control, vol. 46, no. 6, pp. 853-865, Jun. 2001.

[17] M. Staroswiecki, J. P. Cassar, and G. Comtet-Varga, "Analytic redundancy relations for state affine systems," in Proc. 4th Eur. Control Conf., Brussels, Belgium, 1997.

[18] A. B. Trunov and M. M. Polycarpou, "Robust nonlinear fault diagnosis: Application to robotic systems," in Proc. IEEE Int. Conf. Control Applications, Piscataway, NJ, Apr. 1999, pp. 1424-1429.

[19] M. L. Visinsky, J. R. Cavallaro, and I. D. Walker, "Robotic fault detection and fault tolerance: A survey," Reliab. Eng. Syst. Saf., vol. 46, no. 2, pp. 139-158, 1994.

[20] —_ "A dynamic fault tolerance framework for remote robots," IEEE Trans. Robot. Autom., vol. 11, no. 4, pp. 477-490, Aug. 1995.

[21] J. Wünnenberg and P. M. Frank, "Dynamic model based incipient fault detection concept for robots," in Proc. IFAC 11th Triennial World Congr., Tallinn, USSR, Aug. 1990, pp. 61-66.

[22] A. N. Zhirabok and O. V. Preobragenskaya, "Instrument fault detection in nonlinear dynamic systems," in Proc. IEEE Int. Conf. Systems, Man, and Cybernetics, Le Touquet, France, Oct. 1993, pp. 114-119. 